Pacific Journal of Mathematic 


\title{
SOME RESULTS ON PSEUDO-CONTRACTIVE MAPPINGS
}

\author{
W. A. KIRK AND RAINALD SchÖNEBERG
}

Let $E$ be a Banach space and $D$ a subset of $E$. A mapping $f: D \rightarrow E$ such that $\|u-v\| \leqq \|(1+r)(u-v)-r(f(u)-$ $f(v)) \|$ for all $u, v \in D, r>0$ is called pseudo-contractive. The basic result is the following: Let $X$ be a bounded closed subset of $E$, suppose $f: X \rightarrow E$ is a continuous pseudo-contractive mapping such that $f[X]$ is bounded, and suppose there exists $z \in X$ such that $\|z-f(z)\|<\|x-f(x)\|$ for all $x \in$ boundary $(X)$. Then inf $\{\|x-f(x)\|: x \in X\}=0$. If in addition $X$ has the fixed point property with respect to nonexpansive self-mappings, then $f$ has a fixed point in $X$. It follows from this result that if $T: E \rightarrow E$ is continuous and accretive with $\|T(x)\| \rightarrow \infty$ as $\|x\| \rightarrow \infty$, then $T[E]$ is dense in $E$, and if in addition it is assumed that the closed balls in $E$ have the fixed-point property with respect to nonexpansive self-mappings, then $T[E]=E$. Also included are some theorems for continuous pseudo-contractive mappings $f$ which involve demi-closedness of $I-f$ and consequently require uniform convexity of $E$.

1. Introduction. Let $E$ be a Banach space, $X$ a subset of $E$, and $f$ a mapping of $X$ into $E$. Then $f$ is said to be nonexpansive if for all $x, y \in X$,

$$
\|f(x)-f(y)\| \leqq\|x-y\|
$$

while $f$ is said to be pseudo-contractive if for all $x, y \in X$ and $r>0$,

$$
\|x-y\| \leqq\|(1+r)(x-y)-r(f(x)-f(y))\| \text {. }
$$

The pseudo-contractive mappings (which are clearly more general than the nonexpansive mappings) derive their importance in nonlinear functional analysis via their firm connection with the accretive transformations: A mapping $f: X \rightarrow E$ is pseudo-contractive if and only if the mapping $T=I-f$ is accretive, i.e., for every $x, y \in X$ there exists $j \in J(x-y)$ such that

$$
\operatorname{Re}(T(x)-T(y), j) \geqq 0
$$

where $J: E \rightarrow 2^{E^{*}}$ is the normalized duality mapping which is defined by

$$
J(x)=\left\{j \in E^{*}:(x, j)=\|x\|^{2},\|j\|=\|x\|\right\} .
$$

(See Browder [3]; Kato [13].) 
Recent interest in mapping theory for accretive transformations, particularly as it relates to existence theorems for nonlinear differential equations, has prompted a corresponding interest in fixed-point theory for pseudo-contractive mappings (e.g., [2], [7], [8], [13], [18], [21], [23], [26]). This latter theory is intimately connected with the fixed-point theory for nonexpansive mappings. We utilize this fact in the present paper, obtaining in the process new fixed point theorems for continuous pseudo-contractive mappings which are then applied to show (Theorem 3) that if $E$ is a Banach space and $T: E \rightarrow E$ a continuous accretive mapping which satisfies $\|T(x)\| \rightarrow \infty$ as $\|x\| \rightarrow \infty$, then $T[E]$ is dense in $E$, and moreover $T[E]=E$ if it is assumed in addition that the closed balls in $E$ have the fixedpoint property with respect to nonexpansive selfmappings. We conclude with some theorems for continuous pseudo-contractive mappings $f$ which involve demi-closedness of $I-f$ and consequently require the explicit assumption of uniform convexity of the space. We should also mention that our development is structured to reveal the distinction between results obtainable by elementary methods for lipschitzian (or more generally, $k$-set-contractive) mappings and the corresponding sharper results for continuous mappings which are based upon rather deep theorems in differential equations due to Martin [18] and Deimling [8].

Throughout our discussion, $E$ will denote a Banach space, and for $X \subset E$ we use int $(X)$ to denote the interior of $X$ and $\partial X$ to denote the boundary of $X$. By a contraction mapping we shall always mean a mapping with Lipschitz constant strictly less than 1.

We need the following fact for the proof of Theorem 1 .

Proposition 1. Let $X$ be an open subset of a Banach space $E$ and $U: \bar{X} \rightarrow E$ a contraction mapping satisfying for some $z \in X$ the Leray-Schauder boundary condition:

$$
U(x)-z \neq \lambda(x-z) \text { for all } x \in \partial X, \lambda>1 .
$$

Then $U$ has a fixed point in $\bar{X}$.

Proposition 1 is closely related to Theorem 5a of Browder [4]. A degree-theoretic proof for the more general condensing mapping (and bounded $X$ ) is implicit in the development of R. Nussbaum [19] and given explicitly in Petryshyn [20], while an elementary proof of Proposition 1 for contraction mappings (sufficient for our purposes) may be found in Gatica-Kirk [11].

Because we shall frequently refer to results of Deimling [8] for 
strongly accretive mappings we include his definition: Let $D \subset E$. A mapping $T: D \rightarrow E$ is strongly accretive if for each $x, y \in D$,

$$
\sup \{\operatorname{Re}(T(x)-T(y), j): j \in J(x-y)\} \geqq \alpha(\|x-y\|)\|x-y\|
$$

where $\alpha: R^{+} \rightarrow R^{+}$is continuous with $\alpha(0)=0$ and $\alpha(s)>0$ for $s>0$.

2. General results. The results of this section are formulated either in arbitrary Banach spaces or, for stronger conclusions, in spaces in which the domain $X$ of the mapping in question has the fixed-point property relative to nonexpansive self-mappings. The precise generality of the class of sets $X$ satifying this latter condition is not known, but it does include all weakly compact convex sets which possess 'normal structure,' in particular all bounded closed convex subsets of unifomly convex spaces (Browder [1], Göhde [12], Kirk [16]), and in fact Karlovitz [14, 15] has recently discovered special instances in which neither weak compactness nor normal structure is essential for this condition.

THEOREM 1. Let $X$ be a bounded closed subset of a Banach space $E$ (with int $(X) \neq \varnothing$ ). Suppose $f: X \rightarrow E$ is a continuous pseudocontractive mapping and suppose there exists $z \in X$ such that

$$
\|z-f(z)\|<\|x-f(x)\| \text { for all } x \in \partial X .
$$

Then $\inf \{\|x-f(x)\|: x \in X\}=0$. If in addition $X$ has the fixedpoint property with respect to nonexpansive self-mappings, then $f$ has a fixed point in $X$.

Before proving Theorem 1 we state the other results of this section.

Theorem 2. Let $E$ be a Banach space, $f: E \rightarrow E$ a continuous pseudo-contractive mapping and suppose that for some $\delta>0$ the set $\{x \in E:\|x-f(x)\| \leqq \delta\}$ is nonempty and bounded. Then

$$
\inf \{\|x-f(x)\|: x \in E\}=0 \text {. }
$$

If in addition closed balls in $E$ have the fixed-point property with respect to nonexpansive self-mappings, then $f$ has a fixed point in $E$.

THEOREm 3. Let $E$ be a Banach space and $T: E \rightarrow E$ a continuous accretive transformation such that $\|T(x)\| \rightarrow \infty$ as $\|x\| \rightarrow \infty$. Then the range of $T$ is dense in $E$. If in addition closed balls in $E$ have the fixed point property with respect to nonexpansive self-mappings, then the range of $T$ is all of $E$. 
Using an equivalent definition of accretivity (see the remarks below), Deimling has observed (see [8, p. 373]) that the surjectivity portion of the above result holds under the possibly stronger assumption that the closed bounded convex sets in $E$ have the common fixed point property with respect to commuting families of nonexpansive self-mappings. It is known (Bruck [5]) that if such a set $B$ is either weakly compact or separable and if every nonexpansive mapping $f: B \rightarrow B$ has a fixed point in every $f$-invariant nonempty closed convex subset of $B$, then $B$ has this common fixed-point property. As noted above, however, nonweakly compact sets may have the fixed point property for nonexpansive self-mappings. (In fact the proof of [16] can be modified to show that a weak*-compact convex subset of a conjugate space has this property if it possesses normal structure.) Thus while it is not clear to what extent our result improves Deimling's, our method appears to be considerably different in that we avoid completely the use of a common fixed point theorem.

Proof of Theorem 1. We show first that inf $\{\|x-f(x)\|: x \in X\}=$ 0 . Since $f$ is pseudo-contractive we have for fixed $r \in(0,1), u, v \in X$ :

$$
\|u-v\| \leqq r\|u-v\|+\|(I-r f)(u)-(I-r f)(v)\| ;
$$

thus

$$
(1-r)\|u-v\| \leqq\|(I-r f)(u)-(I-r f)(v)\|
$$

and hence the mapping $U=(1-r)(I-r f)^{-1}$ is defined and nonexpansive on $B=(I-r f)[X]$. Moreover from (2) there exists $j \in$ $J(u-v)$ such that

$$
\operatorname{Re}((I-r f)(u)-(I-r f)(v), j) \geqq(1-r)\|u-v\|^{2}
$$

and it follows that $I-r f$ is strongly accretive (with $\alpha(s)=(1-r) s$ ). Thus by Theorem 3 of [8] $(I-r f)[\operatorname{int}(X)]$ is open, while by (3) $B=(I-r f)[X]$ is closed. It follows that $\partial B \subset(I-r f)[\partial X]$. Also, for $\bar{x}=x-r f(x) \in B$ we have

$$
\|\bar{x}-U(\bar{x})\|=r\|x-f(x)\| .
$$

Now let $\bar{x} \in \partial B$ and $\bar{z}=z-r f(z)$ where $z \in X$ is the point specified in the statement of the theorem. Since $\|z-f(z)\|<\|x-f(x)\|$ it follows that

$$
\|\bar{z}-U(\bar{z})\|<\|\bar{x}-U(\bar{x})\| \text {. }
$$

The assumption $U(\bar{x})-\bar{z}=\lambda(\bar{x}-\bar{z})$ for $\lambda>1$ leads to a contradiction because it implies $\|U(\bar{x})-\bar{z}\|=\lambda\|\bar{x}-\bar{z}\|$ and 


$$
\|\bar{x}-U(\bar{x})\|=(\lambda-1)\|\bar{x}-\bar{z}\|
$$

while (5), the nonexpansiveness of $U$ and $\bar{x} \neq \bar{z}$ yields

$$
\begin{aligned}
\|U(\bar{x})-\bar{z}\| & \leqq\|U(\bar{x})-U(\bar{z})\|+\|U(\bar{z})-\bar{z}\| \\
& <\|\bar{x}-\bar{z}\|+\|\bar{x}-U(\bar{x})\| ;
\end{aligned}
$$

i.e., $\lambda\|\bar{x}-\bar{z}\|<\|\bar{x}-\bar{z}\|+(\lambda-1)\|\bar{x}-\bar{z}\|$, a contradiction. We thus conclude:

$$
U(\bar{x})-\bar{z} \neq \lambda(\bar{x}-\bar{z}) \text { for all } \bar{x} \in \partial B \text { and } \lambda>1 .
$$

It follows that for $t \in(0,1)$ the mapping $U_{t}: B \rightarrow E$ defined by

$$
U_{t}(\bar{x})=(1-t) \bar{z}+t U(\bar{x}), \quad \bar{x} \in B,
$$

is a contraction mapping which satisfies the Leray-Schauder condition:

$$
U_{t}(\bar{x})-\bar{z} \neq \lambda(\bar{x}-\bar{z}) \text { for all } \bar{x} \in \partial B, \quad \lambda>1 .
$$

By Proposition 1, $U_{t}$ has a fixed point $\bar{x}_{t} \in B$; thus

$$
\begin{aligned}
\left\|\bar{x}_{t}-U\left(\bar{x}_{t}\right)\right\| & =\left\|(1-t) \bar{z}+t U\left(\bar{x}_{t}\right)-U\left(\bar{x}_{t}\right)\right\| \\
& \leqq(1-t)\left[\|\bar{z}\|+\left\|U\left(\bar{x}_{t}\right)\right\|\right] .
\end{aligned}
$$

Because $U$ maps $B$ into $(1-r) X$ and the latter set is bounded it follows that $\left\{U\left(\bar{x}_{t}\right)\right\}$ is bounded and thus (7) implies inf $\{\|\bar{x}-U(\bar{x})\|: \bar{x} \in B\}=0$. The first part of the theorem now follows from (4).

We now prove existence of a fixed point of $f$ with the added hypothesis that any nonexpansive mapping of $X$ into $X$ always has a fixed point. First, notice that in view of the fact that

$$
\inf \{\|x-f(x)\|: x \in X\}=0
$$

we may assume existence of $z \in X$ such that

$$
\|z-f(z)\|<\inf \{\|x-f(x)\|: x \in \partial X\} \text {. }
$$

Since $X$ is bounded, (8) implies $\alpha \in(0,1)$ may be chosen so near 1 that for all $y \in X$,

$$
\begin{aligned}
& \alpha\|z-f(z)\|+(1-\alpha)\|z-y\| \\
& \quad<\inf \{\alpha\|x-f(x)\|-(1-\alpha)\|x-y\|: x \in \partial X\} .
\end{aligned}
$$

Now define $U_{\alpha, y}: X \rightarrow E$ by

$$
U_{\alpha, y}(x)=(1-\alpha) y+\alpha f(x), \quad x \in X .
$$

(I). Suppose it is the case that for fixed $\alpha \in(0,1), U_{\alpha, y}$ has a 
fixed point $F_{\alpha}(y)$ for each $y \in X$.

Then a mapping $F_{\alpha}: X \rightarrow X$ can be defined with the property

$$
F_{\alpha}(y)=(1-\alpha) y+\alpha f\left(F_{\alpha}(y)\right), \quad y \in X .
$$

Thus for $u, v \in X$,

$$
F_{\alpha}(u)-F_{\alpha}(v)=\alpha\left(f\left(F_{\alpha}(u)\right)-f\left(F_{\alpha}(v)\right)\right)+(1-\alpha)(u-v) ;
$$

and for $j \in J\left(F_{\alpha}(u)-F_{\alpha}(v)\right)$,

$$
\begin{aligned}
& \left(F_{\alpha}(u)-F_{\alpha}(v), j\right) \\
& \left.\quad=\alpha\left(f_{\alpha}(u)\right)-f\left(F_{\alpha}(v)\right), j\right)+(1-\alpha)((u-v), j) .
\end{aligned}
$$

Hence for suitable such $j$ we have by (2):

$$
\begin{aligned}
& \left\|F_{\alpha}(u)-F_{\alpha}(v)\right\|^{2} \\
& \quad \leqq \alpha\left\|F_{\alpha}(u)-F_{\alpha}(v)\right\|^{2}+(1-\alpha)\|u-v\|\left\|F_{\alpha}(u)-F_{\alpha}(v)\right\|,
\end{aligned}
$$

i.e.,

$$
\left\|F_{\alpha}(u)-F_{\alpha}(v)\right\| \leqq\|u-v\| \text {. }
$$

Therefore $F_{\alpha}$ is a nonexpansive mapping of $X$ into $X$ and since $F_{\alpha}(x)=x$ only if $f(x)=x$, under our added hypothesis on $X$ we need only establish (I) to complete the proof of the theorem.

Returning to (9) and the definition (10) of $U_{\alpha, y}$ we have

$$
\left\|z-U_{\alpha, y}(z)\right\|<\inf \left\{\left\|x-U_{\alpha, y}(x)\right\|: x \in \partial X\right\} .
$$

Fix $y \in X$ and with $r$ chosen in $(0,1)$, let $S=I-r U_{\alpha, y}$. Then for $u, v \in X$ and appropriate $j \in J(u-v)$ we have by pseudo-contractiveness of $f$ and (2),

$$
\begin{aligned}
\operatorname{Re}(S(u)-S(v), j) & =\operatorname{Re}(u-r \alpha f(u)-(v-r \alpha f(v)), j) \\
& =\|u-v\|^{2}-r \alpha \operatorname{Re}(f(u)-f(v), j) \\
& \geqq(1-\alpha r)\|u-v\|^{2} ;
\end{aligned}
$$

i.e., $S$ is strongly accretive and by Theorem 3 of [8] $S[\operatorname{int}(X)]$ is open. Hence $S(z) \in \operatorname{int}(X)$ and since $S[X]$ is closed, $\partial(S[X]) \subset S[\partial X]$. We next show that if $H=(1-r) S^{-1}$ then

(i) $H$ is a contraction mapping, and

(ii) $H$ satisfies the Leray-Schauder boundary condition: $H(\bar{x})-$ $\bar{z} \neq \lambda(\bar{x}-\bar{z})$ for $\bar{x} \in \partial D$ and $\lambda>1$ where $D=S[X]$ and $\bar{z}=S(z)$.

To prove (i) notice that by (12),

$$
(1-\alpha r)\|u-v\| \leqq\|S(u)-S(v)\|, \quad u, v \in X,
$$

from which 


$$
\|H(s)-H(t)\| \leqq\left(\frac{1-r}{1-\alpha r}\right)\|s-t\|, \quad s, t \in D .
$$

To prove (ii) observe that $\|\bar{z}-H(\bar{z})\|=r\left\|z-U_{\alpha, y}(z)\right\|$. Now let $\bar{x} \in \partial D$ where $\bar{x}=x-r U_{\alpha, y}(x)$. Then $\|\bar{x}-H(\bar{x})\|=r\left\|x-U_{\alpha, y}(x)\right\|$ and since $x \in \partial X$ (recall, $\partial D \subset S[\partial X]$ ) we have by (11)

$$
\|\bar{z}-H(\bar{z})\|<\|\bar{x}-H(\bar{x})\| \text {. }
$$

The assumption that $H(\bar{x})-\bar{z}=\lambda(\bar{x}-\bar{z})$ for $\lambda>1$ now leads to a contradiction in the same manner as in the proof of the first part of the theorem for the mapping $U$.

Having established (i) and (ii), $H$ has a fixed point $\bar{w} \in D$ by Proposition 1. From this,

$$
(1-r)\left(I-r U_{\alpha, y}\right)^{-1}(\bar{w})=\bar{w} ;
$$

hence

$$
\frac{\bar{w}}{1-r}-r U_{\alpha, y}\left(\frac{\bar{w}}{1-r}\right)=\bar{w}
$$

which in turn implies $U_{\alpha, y}(\bar{w} /(1-r))=\bar{w} /(1-r)$, proving (I) and completing the proof of Theorem 1 .

We use the following lemma (cf. [25]) in the proof of Theorem 2 and include its proof for the sake of completeness.

LeMma 1. Let $X$ be a subset of a Banach space $E$ and let $f: X \rightarrow E$ be a continuous pseudo-contractive mapping. If $A_{f}: X \rightarrow E$ is defined by $A_{f}=2 I-f$, then:

(a) $A_{f}$ is one-to-one and $A_{f}^{-1}$ is nonexpansive.

(b) $f$ and $A_{f}^{-1}$ have the same fixed points.

(c) If $X$ is closed, $A_{f}[X]$ is closed.

(d) If $X$ is open, then $A_{f}[X]$ is open.

Proof. (a), (c): We have by definition (taking $r=1$ ),

$$
\left\|A_{f}(x)-A_{f}(y)\right\| \geqq\|x-y\| \text {. }
$$

(b): Obvious. (d): Let $x, y \in X$ and choose $j \in J(x-y)$ so that

$$
\operatorname{Re}(f(x)-f(y), j) \leqq\|x-y\|^{2} .
$$

Thus

$$
\begin{aligned}
\operatorname{Re}\left(A_{f}(x)-A_{f}(y), j\right) & =2(x-y, j)-\operatorname{Re}(f(x)-f(y), j) \\
& \geqq\|x-y\|^{2} ;
\end{aligned}
$$


thus $A_{f}$ is strongly accretive and $A_{f}[X]$ is open by [8, Theorem 3].

Proof of Theorem 2. Since $A_{f}[E]=E$ (by Lemma 1, (c)-(d)) we may define $g: E \rightarrow E$ by $g=A_{f}^{-1}$. Then $g$ is nonexpansive. Let $D=$ $\{x \in E:\|x-f(x)\| \leqq \delta\}$ and choose $y \in D$. Since $D$ is bounded so is $A_{f}[D]$; hence there is a ball $B$ such that $A_{f}[D] \subset \operatorname{int}(B)$. Set $z=A_{f}(y)$. For $x \in \partial B$ we have $\|z-g(z)\|=\|y-f(y)\| \leqq \delta<\|g(x)-f(g(x))\|=$ $\|x-g(x)\|$. Theorem 2 now follows from Theorem 1 and Lemma 1(b).

Proof of Theorem 3. Let $z \in E$ and $f=I-T+z$. Then $f$ is a continuous pseudo-contractive mapping and if $\delta>0,\|x-f(x)\| \leqq \delta$ implies $\|T(x)\| \leqq \delta+\|z\|$. Thus for $\delta$ sufficiently large the set $\{x \in E:\|x-f(x)\| \leqq \delta\}$ is nonempty and bounded; hence inf $\{\|x-f(x)\|:$ $x \in E\}=0$ by Theorem 2 yielding $z \in \overline{f(E)}$. If closed balls in $E$ have the fixed point property with respect to nonexpansive self-mappings Theorem 2 yields $x \in E$ such that $x=f(x)$ from which $T(x)=z$.

3. Uniformly convex spaces. With $E$ uniformly convex, $K$ a closed convex subset of $E$, and $f: K \rightarrow E$ nonexpansive, then $I-f$ is demi-closed on $K$, i.e., if $x_{n}-f\left(x_{n}\right) \rightarrow y$ strongly for $\left\{x_{n}\right\} \subset K$ while $x_{n} \rightarrow x$ weakly, then $x-f(x)=y$. This important property of nonexpansive mappings is implicit in Göhde [12] and an explicit proof based upon Göhde's technique is given by Browder [4, Theorem 3]. Its application is crucial to Theorem 5 of this section. First, however, we prove a result for a more general class of spaces.

THEOREM 4. Suppose $E$ is a reflexive Banach space such that every nonempty closed bounded and convex subset of $E$ has the fixed point property with respect to nonexpansive selfmappings and suppose $f: E \rightarrow E$ is a continuous pseudo-contractive mapping. If $x_{n}-f\left(x_{n}\right) \rightarrow 0$ strongly for some bounded sequence $\left\{x_{n}\right\} \subset E$, then $f$ has a fixed point.

Proof. By Lemma 1, $A_{f}[E]=E$ (where $\left.A_{f}=2 I-f\right)$. Let $g=$ $A_{f}^{-1}$ and $y_{n}=A_{f}\left(x_{n}\right)$. Then $\left\{y_{n}\right\}$ is bounded and moreover $y_{n}-g\left(y_{n}\right)=$ $x_{n}-f\left(x_{n}\right) \rightarrow 0$ strongly. Let $C$ denote the set of asymptotic centers of $\left\{y_{n}\right\}$ (cf. Edelstein [9]). Then $C$ is nonempty, closed, bounded and convex and since $g$ is nonexpansive, $g$ maps $C$ into $C$ (see Reich [22]). Thus $g$ has a fixed point by assumption. Lemma 1(b) finishes the proof.

THEOREM 5. Let $E$ be a uniformly convex Banach space, $X$ a bounded closed convex subset of $E$, and $G$ an open set containing $X$ with $\operatorname{dist}(X, E \backslash G)>0$. Suppose $f: \bar{G} \rightarrow E$ is a continuous pseudo- 
contractive mapping which sends bounded sets into bounded sets. Then $I-f$ is demi-closed on $X$.

Proof. Suppose $\left\{x_{n}\right\} \subset X$ with $x_{n}-f\left(x_{n}\right) \rightarrow y$ strongly while $x_{n} \rightarrow x_{0}$ weakly. We must show $x_{0}-f\left(x_{0}\right)=y$ and clearly (replacing $f$ with $f+y$ ) we may assume $y=0$. Since $X$ is bounded and convex we may suppose that $G$ is bounded and convex with $\delta=\operatorname{dist}(X, E \backslash G)>0$. Let $\tilde{X}$ be a closed $\delta / 2$-neighborhood of $X$. It is possible to choose $r \in(0,1)$ small enough that (i) for each $z \in \tilde{X}$ and $y \in \bar{G}, z+r f(y) \in \bar{G}$, and (ii) $x_{n}-r f\left(x_{n}\right) \in \tilde{X}$. Then the mapping $U_{r, z}: \bar{G} \rightarrow E$ defined by

$$
U_{r, z}(y)=z+r f(y)
$$

maps $\bar{G}$ into $\bar{G}$. Observing (2) it follows from Corollary 2 of [8] that for each $z \in \tilde{X}$ there exists $y_{z} \in \bar{G}$ such that $U_{r, z}\left(y_{z}\right)=y_{z}$ Hence $(I-r z)\left(y_{z}\right)=z$ and this proves that $\tilde{X}$ lies in $(I-r f)[\tilde{G}]$. The mapping $H=(i-r)(1-r f)^{-1}$ is nonexpansive (cf. (3)) and defined on $(I-r f)[\bar{G}]$. Moreover if $\bar{x}_{n}=x_{n}-r f\left(x_{n}\right)$, then $\bar{x}_{n}-H\left(\bar{x}_{n}\right)=$ $r\left(x_{n}-f\left(x_{n}\right)\right) \rightarrow 0$ strongly while $x_{n} \rightarrow(1-r) x_{0}$ weakly. By (ii) the sequence $\left\{\bar{x}_{n}\right\}$ lies in $\tilde{X}$ and by demi-closedness of $H$ on $\tilde{X},(1-r) x_{0}=$ $H\left((1-r) x_{0}\right)$ from which $x_{0}=f\left(x_{0}\right)$.

THEOREM 6. Let $E$ be a uniformly convex Banach space, $X$ a bounded closed convex subset of $E$ with int $(X) \neq \varnothing$, and $G$ an open set containing $X$ such that $\operatorname{dist}(X, E \backslash G)>0$. Suppose $f: G \rightarrow E$ is a continuous pseudo-contractive mapping which sends bounded sets into bounded sets and satisfies for some $z \in \operatorname{int}(X)$ :

$$
f(x)-z \neq \lambda(x-z) \text { for } \quad x \in \partial X, \quad \lambda>1 .
$$

Then $f$ has a fixed point in $X$.

Proof. By replacing $f(x)$ with $f(x-z)+z$ one may take $z=0$ in $\left({ }^{*}\right)$ (and thus by assumption $0 \in \operatorname{int}(X)$ ). For $r \in(0,1)$, the mapping $T=I-r f$ is strongly accretive and by [8, Theorem 3] $T$ [int $(X)$ ] is open. As we have seen earlier $T[X]$ is closed and thus $\partial T[X] \subset$ $T[\partial X]$. Since $f[X]$ is bounded it is possible to choose $r \in(0,1)$ so small that $r f[X] \subset \operatorname{int}(X)$ and thus by [8, Corollary 2] we have $0 \in$ $T[\operatorname{int}(X)] \subset \operatorname{int}(T[X])$.

By Theorem 5, $I-f$ is demi-closed on $X$ and since $X$ is weakly compact, $(I-f)[X]$ is closed. With this and the observations above, it is possible to follow precisely the argument of Gatica-Kirk [10, p. 113] (letting $f$ play the role of $U$ ) to show that for the nonexpansive mapping $H=(1-r) T^{-1}: T[X] \rightarrow E,(I-H)[T[X]]$ is closed and $H$ satisfies the Leray-Schauder boundary condition: 


\section{$H(x) \neq \lambda x$ for $x \in \partial T[X]$ and $\lambda>1$.}

Since $H$ is nonexpansive a routine application of Proposition 1 (to mappings $t H, t \in(0,1)$ yields inf $\{\|x-H(x)\| x \in T[X]\}=0$ and with $(I-H)[T[X]]$ closed it follows that $H$, hence $f$, has a fixed point.

Finally, we observe that a slight modification of a portion of the above argument yields a result for arbitrary spaces.

TheOREM 7. Let $E$ be a Banach space, $X$ a closed bounded and convex subset of $E$ with int $(X) \neq \varnothing$ and $f: X \rightarrow E$ a continuous pseudo-contractive mapping such that $f[X]$ is bounded. Suppose there exists $z \in \operatorname{int}(X)$ such that

$$
f(x)-z \neq \lambda(x-z) \text { for } \quad x \in \partial X, \quad \lambda>1 .
$$

Then $\inf \{\|x-f(x)\|: x \in X\}=0$.

Proof. As before, by replacing $f(x)$ with $f(x+z)-z$ and $X$ by $X-z$, one may take $z=0$ in $\left(^{*}\right)$ (and thus $0 \in \operatorname{int}(X)$ ). Choose $r>0$ such that $r(1+r)^{-1} f[X] \subset \operatorname{int}(X)$ and let $T=(1+r) I-r f$. Then since $I-f$ is accretive, $T$ is strongly accretive; hence $T[\operatorname{int}(X)]$ is open by [8, Theorem 3]. As we have seen earlier, $T[X]$ is closed. Thus $\partial D \subset T[\partial X]$ where $D=T[X]$. The mapping $g: D \rightarrow E$ de fined by $g=T^{-1}$ is nonexpansive. Since $\|y-g(y)\|=$ $r\|g(y)-f(g(y))\|$ for $y \in D$, by Proposition 1 it suffices to show that $0 \in \operatorname{int}(D)$ and that $g(y) \neq \lambda y$ for $y \in \partial D$ and $\lambda>1$. Using $r(1+r)^{-1} f[X] \subset \operatorname{int}(X)$, [8, Corollary 2] implies the existence of $x_{0} \in \operatorname{int}(X)$ such that $x_{0}=r(1+r)^{-1} f\left(x_{0}\right)$. Thus $0=T\left(x_{0}\right) \in T[\operatorname{int}(X)] \subset$ int $(D)$. Now suppose $g(y)=\lambda y$ where $y \in \partial D$ and $\lambda>1$. Choose $x \in \partial X$ such that $T(x)=y$. Then $x=g(y)=\lambda y=\lambda((1+r) x-r f(x))$, i.e., $f(x)=(\lambda(1+r)-1) /(r \lambda) x$, and since $\lambda(1+r)-1>r \lambda$, this contradicts $(*)$.

REMARKS. If $f$ is assumed to be lipschitzian in Theorem 1 then $r>0$ can be chosen so small that $r f$ is a contraction mapping and it follows (as is well-known and easily proved) that $(I-r f)[X]$ is closed and $(I-r f)[\operatorname{int}(X)]$ is open. This renders appeal to [8, Theorem 3] unnecessary. Similar reasoning applies throughout and in fact it is possible (as seen in an earlier version of this paper) to obtain all our results by elementary direct methods if all the mappings considered are assumed to be 'lipschitzian' rather than 'continuous.' We comment on this because the extent to which results of this type are obtainable without appeal to existence theorems for differential equations has been a topic of recent interest ([6], [22]), and we know of no elementary proofs for the more general versions of our 
theorems.

We should also add that in the proof of Theorem 1 the nonexpansiveness of the mapping $F_{\alpha}$ was originally brought to our attention by R. E. Bruck, Jr. Also the observation that the definition of accretivity used in [8] is equivalent to the usual one (used here) was brought to our attention by Juan A. Gatica. This latter fact follows easily from the weak*-compactness of closed balls in $X^{*}$.

\section{REFERENCES}

1. F. E. Browder, Nonexpansive nonlinear operators in a Banach space, Proc. Nat. Acad. Sci, U.S. A., 53 (1965), 1272-1276.

2. - Nonlinear equations of evolution and nonlinear accretive operators in Banach spaces, Bull. Amer. Math. Soc., 73 (1967), 867-874.

3. - Nonlinear mappings of nonexpansive and accretive type in Banach spaces, Bull. Amer. Math. Soc., 73 (1967), 875-882.

4. - Semicontractive and semiaccretive nonlinear mappings in Banach spaces, Bull. Amer. Soc., 74 (1968), 660-665.

5. R. E. Bruck, Jr., A common fixed point theorm for a commuting family of nonexpansive mappings, Pacific J. Math., 53 (1974), 59-71.

6. J. Caristi, Fixed point theorems for mappings satsfying inwardness conditions, Trans. Amer. Math. Soc., 215 (1976), 241-251.

7. M. G. Crandall, Differential equations on convex sets, J. Math. Soc. Japan, 22 (1970), 443-455.

8. K. Deimling, Zeros of accretive operators, Manuscripta Math., 13 (1974), 365-374.

9. M. Edelstein, The construction of an asymptotic center with a fixed point property, Bull. Amer. Math. Soc., 78 (1972), 206-208.

10. J. A. Gatica and W. A. Kirk, Fixed point theorems for lipschitzian pseudo-contractive mappings, Proc. Amer. Math. Soc., 36 (1972), 111-115.

11. —- Fixed point theorems for contration mappings with applications to nonexpansive and pseudo-contractive mappings, Rocky Mountain J. Math., 4 (1974), 69-79.

12. D. Göhde, Zum Prinzip der kontraktiven Abbildung, Math. Nachr., 30 (1965), 251-258.

13. T. Kato, Nonlinear semigroups and evolution equations, J. Math. Soc. Japan, 19 (1967), 508-520.

14. L. A. Karlovitz, Some fixed point results for nonexpansive mappings, Proceedings of a Seminar on Fixed Point Theory and its Applications, Dalhousie University, Halifax, N. S., June 9-12 (1975), Academic Press, (1976), 91-103.

15. - Existence of fixed points of nonexpansive mappings in a space without normal structure, (to appear).

16. W. A. Kirk, A fixed point theorem for mappings which do not increase distances, Amer. Math. Monthly, 72 (1965), 1004-1006.

17. - Remarks on pseudo-contractive mappings, Proc. Amer. Math. Soc., 25 (1970), 820-823.

18. R. H. Martin, Differential equations on closed subsets of a Banach space, Trans. Amer. Math. Soc., 179 (1973), 399-414.

19. R. Nussbaum, The fixed point index for local condensing mappings, Ann. Mat. Pura Appl., (4), 89 (1971), 217-258.

20. W. V. Petryshyn, Structure of the fixed point set of k-set-contractions, Arch. Rational Mech. Anal., 40 (1970/71), 312-328.

21. - Remarks on condensing and k-set-contractive mappings, J. Math. Anal. Appl., 39 (1972), 717-741. 
22. S. Reich, Remarks on fixed points II, Atti. Lincei. Rend. Cd. Sci. Fis. Mat., 53 (1972), 250-254.

23. - On fixed point theorems obtained from existence theorems for differential equations, J. Math. Anal. Appl., 54 (1976), 26-36.

24. J. Reinermann and $\mathrm{R}$. Schöneberg, Some results and problems in the fixed point theory for nonexpansive and pseudocontractive mappings in Hilbert space, Proceedings of a Seminar on Fixed Point Theory and its Applications, Dalhousie University, Halifax, N.S., June 9-12 (1975), Academic Press, (1976), 187-196.

25. R. Schöneberg, On the structure of fixed point sets of pseudo-contractive mappings, Comment. Math. Univ. Car., (to appear).

26. G. Vidossich, How to get zeros of nonlinear operators using the theory of ordinary differential equations, Separata das Atas da Semana de Análise Funcional não linearvol. 5 .

Received March 3, 1975 and in revised form October 15, 1976. Research by the first author was supported in part by National Science Foundation grants, GP 18045 and MCS 76-03945. Part of this work was carried out while the first author was Visiting Professor at the University of British Columbia.

UNIVERSITY OF IOWA

IOWA CITY, IA 52242

AND

TeChnische Hochschule AaChen 


\section{PACIFIC JOURNAL OF MATHEMATICS}

\section{EDITORS}

RICHARD ARENS (Managing Editor)

University of California

Los Angeles, California 90024

C. W. CURTIS

University of Oregon

Eugene, OR 97403

C. C. MOORE

University of California

Berkeley, CA 94720

\section{J. DugundJI}

Department of Mathematics University of Southern Californıa Los Angeles, California 90007

R. FinN AND J. Milgram Stanford University Stanford, California 94305

\section{ASSOCIATE EDITORS}

E. F. BECKENBACH

B. H. NEUMANN

F. WOLF

K. YOSHIDA

\section{SUPPORTING INSTITUTIONS}

UNIVERSITY OF BRITISH COLUMBIA CALIFORNIA INSTITUTE OF TECHNOLOGY UNIVERSITY OF CALIFORNIA MONTANA STATE UNIVERSITY UNIVERSITY OF NEVADA, RENO NEW MEXICO STATE UNIVERSITY OREGON STATE UNIVERSITY UNIVERSITY OF OREGON OSAKA UNIVERSITY
UNIVERSITY OF SOUTHERN CALIFORNIA STANFORD UNIVERSITY UNIVERSITY OF TOKYO UNIVERSITY OF UTAH WASHINGTON STATE UNIVERSITY UNIVERSITY OF WASHINGTON AMERICAN MATHEMATICAL SOCIETY NAVAL WEAPONS CENTER 


\section{Pacific Journal of Mathematics}

\section{Vol. 71, No. $1 \quad$ November, 1977}

Charalambos D. Aliprantis and Owen Sidney Burkinshaw, On universally complete Riesz spaces ............................. 1

Stephen Richard Bernfeld and Jagdish Chandra, Minimal and maximal solutions of nonlinear boundary value problems .................

John H. E. Cohn, The length of the period of the simple continued fraction of

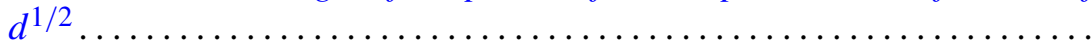

Earl Vern Dudley, Sidon sets associated with a closed subset of a compact abelian group .................................... 33

Larry Finkelstein, Finite groups with a standard component of type $J_{4} \ldots \ldots$

Louise Hay, Alfred Berry Manaster and Joseph Goeffrey Rosenstein, Concerning partial recursive similarity transformations of linearly ordered sets .......................................

Richard Michael Kane, On loop spaces without $p$ torsion. II ............

William A. Kirk and Rainald Schoneberg, Some results on

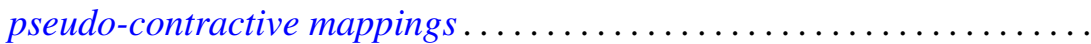

Philip A. Leonard and Kenneth S. Williams, The quadratic and quartic character of certain quadratic units. I. .

Lawrence Carlton Moore, A comparison of the relative uniform topology and the norm topology in a normed Riesz space .................

Mario Petrich, Maximal submonoids of the translational hull 119

Mark Bernard Ramras, Constructing new R-sequences . . .

Dave Riffelmacher, Multiplication alteration and related rigidity properties

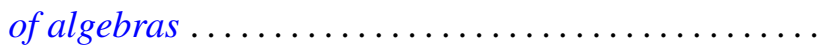

Jan Rosiński and Wojbor Woyczynski, Weakly orthogonally additive functionals, white noise integrals and linear Gaussian stochastic processes.

Ryōtarō Satō, Invariant measures for ergodic semigroups of operators

Peter John Slater and William Yslas Vélez, Permutations of the positive integers with restrictions on the sequence of differences...

Edith Twining Stevenson, Integral representations of algebraic cohomology classes on hypersurfaces ........................

Laif Swanson, Generators of factors of Bernoulli shifts . 